\title{
X-RAY HEATED ACCRETION DISCS AROUND STELLAR MASS BLACK HOLES
}

\author{
Ivan Hubeny ${ }^{1}$ and Dayal T. Wickramasinghe ${ }^{2}$
}

We investigate the effects of irradiation on the vertical structure of accretion discs around black holes and its impact on the emergent energy distribution. Models are presented for a 10 Solar mass black hole in a low mass $\mathrm{X}$-ray binary assuming a black body spectrum for the incident radiation. We show that for a disc annulus at a given radius, the spectra become increasingly distorted as the incident flux increases relative to the viscously generated heating flux in the disc. Significant effects are apparent for rings even at distances of $\sim 10,000$ Schwarzschild radii from the black hole for realistic dilution factors.

$\mathrm{X}$-ray heating is known to play a dominant role in accretion discs around Black Holes in Low Mass Xray Binaries (BH-LMXB). Heating could affect the thermal intability of the disc (El Khoury and Wickramasinghe 1999), and also the emergent spectrum. Of particular interest is the heating that occurs of the outer regions of the disc by radiation that is emitted close to the central black hole. Such an irradiating flux could originate from the hotter central regions of the disc, or from the jet that emanates from the vicinity of the black hole.

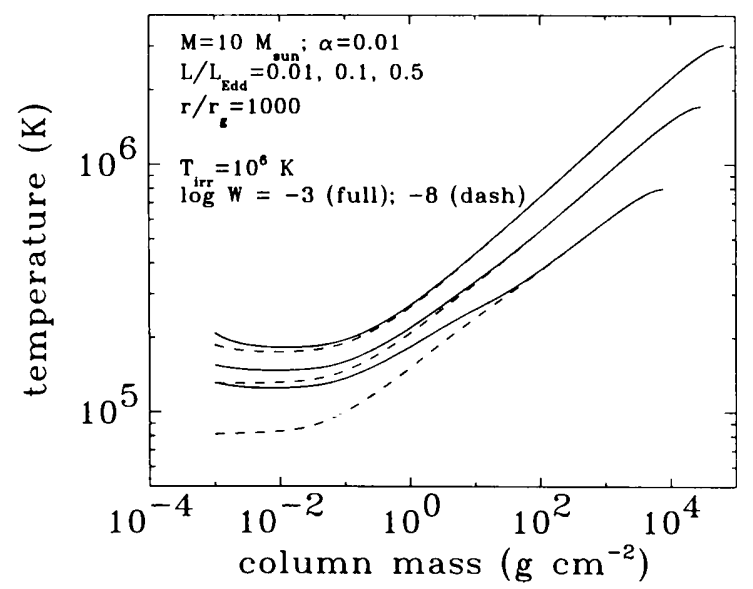

Fig. 1. Spectra of a ring at 1000 gravitational radii irradiated by a one million degree black body for different dilution factors $W$

\footnotetext{
${ }^{1}$ NOAO, Tucson, Arizona, USA

${ }^{2}$ The Australian National University, Canberra. email: dayal@maths.anu.edu.au
}

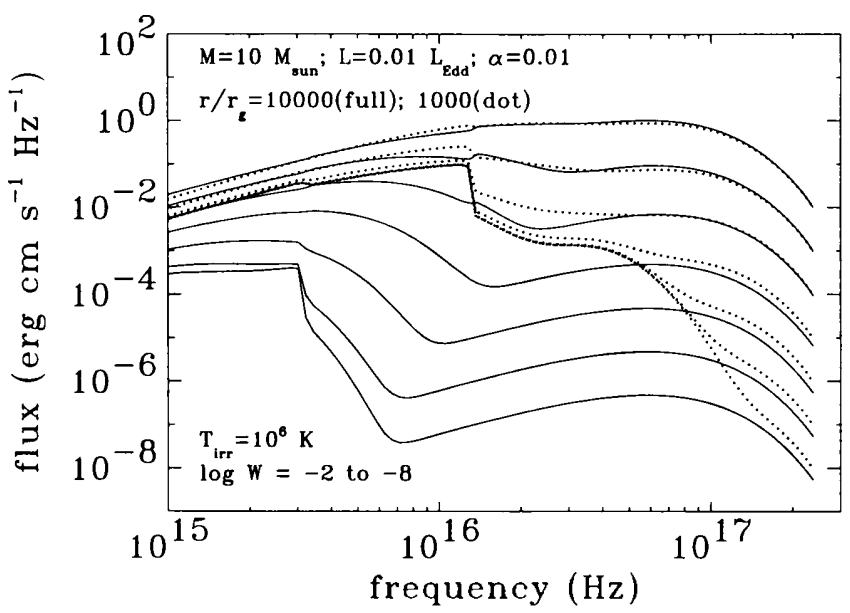

Fig. 2. comparison of spectra of rings at 1000 and 10000 gravitational radii irradiated by a ten million degree black body for different dilution factors $W$

We construct self-consistent vertical structures of disc annuli that allow for external heating. The structures are calculated using the full radiative transfer equations and go beyond previous calculations based on the diffusion approximation (Dubus et al. 1999). The source of irradiation is assumed to be a point source located at the centre of the disc. This assumption is valid provided we only consider disc annuli that are sufficiently far away from the inner regions of the disc. The irradiation is assumed to be isotropic (which may not be a serious limitation), and is included in the calculations as an external boundary condition. We allow for x-ray continuum opacities, and for Compton scattering within an angle-dependent Kompaneets-type approach following Hubeny et al. (2001). In the present illustrative calculations, the spectrum of the incident radiation is assumed to be a black body of temperature $T_{i r r}$ so that we are considering only the case of illumination from the central regions of the disc. The radiation that impinges on the surface of the disc will be diluted by varying amounts depending on the distance from the radiating source and shadowing. This is parametrised by the dilution factor $W$ that appears in our results.

We first illustrate in figure 1 the effects of heating on the vertical temperature structure of a ring 
located at a radial distance of $1000 r_{g}$ in a disc around a Schwarzschild black hole of mass $10 M_{\text {sun }}$. The viscosity parameter is assumed to be $\alpha=0.01$ and we assume that the material flows through the accretion disc at $0.01,0.1$ and 0.6 of the Eddington rate.

The heating effect depends strongly on the incident flux via the dilution factor, and the mass transfer rate through the disc. It is only when this flux is comparable to the viscously generated flux within the ring that heating occurs at depth in the disc. As expected, with the other parameters fixed, the heating effect is strongest for the lowest mass transfer rate. Furthermore, for a fixed mass transfer rate, the heating increases as the dilution decreases (or $W$ increases).

We compare in figure 2 theoretical spectra of two rings located at $1000 r_{g}$ and $10000 r_{g}$ for an accretion rate of .01 the Eddington value. The unirradiated effective temperatures for these two rings are $117,000 \mathrm{~K}$.and $21,000 \mathrm{~K}$ respectively. The ring that is further away from the centre is intrinsically cooler and shows larger heating effects for the same dilution factor $W$. The spectral appearence is determined by a combination true absorption (and thermal re-emission) and compton scattering. The high frequency bump seen in the figures is determined mainly by Compton scattering. As the temperature is increased, this bump moves to higher frequencies reflecting the spectral distribution of the incident flux. We note that the effects of heating are apparent in the overall spectrum well before the irradiation flux becomes comparable to the viscously generated flux because the two components peak at different frequencies, and scattering plays a dominant role at high frequencies.

In order to model observed spectra of black hole transients using our models, one needs to specify the mass transfer rate as a function of radius, and to calculate the dilution factor in a self consistent manner allowing for shadowing. Since the steady disc assumption may not be appropriate for discs in these systems, unstable disc structures will need to be considered. Results of such calculations will be presented in a forthcoming paper.

\section{REFERENCES}

El Khoury, W. \& Wickramasinghe. D. T., 1999, MNRAS, 303. 380

Dubus, S., Lasota, J. P., Hameury, J. M., \&Charles, P., 1999, MNRAS, 139

Hubeny, I., Blaes, O., Krolik, J.,\& Agol, E.,2001, Ap. J., 559,680 